\title{
The Physical and Magnetic Properties of Electrodeposited Co-Fe Nanocoating with Different Deposition Times
}

\author{
Koay Mei Hyie, ${ }^{1}$ Wan Normimi Roslini Abdullah, ${ }^{1}$ Nor Azrina Resali, ${ }^{1}$ \\ W. T. Chong, ${ }^{2}$ Z. Salleh, ${ }^{1}$ and M. A. A. Ghani ${ }^{1}$ \\ ${ }^{1}$ Faculty of Mechanical Engineering, Universiti Teknologi MARA, Selangor, 40450 Shah Alam, Malaysia \\ ${ }^{2}$ Department of Mechanical Engineering, Faculty of Engineering, University of Malaya, 50603 Kuala Lumpur, Malaysia
}

Correspondence should be addressed to Koay Mei Hyie; hyie1105@yahoo.com

Received 11 June 2012; Revised 15 December 2012; Accepted 19 December 2012

Academic Editor: Weichang Hao

Copyright (C) 2013 Koay Mei Hyie et al. This is an open access article distributed under the Creative Commons Attribution License, which permits unrestricted use, distribution, and reproduction in any medium, provided the original work is properly cited.

Using the electrodeposition process, cobalt-iron (Co-Fe) nanocrystalline coatings were successfully synthesized onto stainless steel in deposition times of 30,60, and 90 minutes. The temperature used throughout the process was $50^{\circ} \mathrm{C}$ in an acidic environment of $\mathrm{pH}$ 3. By changing the deposition time, physical properties such as phase and crystallographic structure, surface morphology, grain size, microhardness, and magnetic properties of Co-Fe coatings were examined. FESEM micrographs showed that the grain sizes of the coatings were in the range from $57.9 \mathrm{~nm}$ to $70.2 \mathrm{~nm}$. Dendrite and irregular shapes were found in the microstructure of Co-Fe nanocoating. The Co-Fe nanocrystalline coating prepared in a deposition time of 90 minutes achieved the highest microhardness of $339 \mathrm{HVN}$. The magnetic properties associated with Co-Fe nanocoating at longer deposition times show greater coercivity, $H_{c}$, and saturation magnetization, $M_{s}$, values of $56.43 \mathrm{Oe}$ and $70.45 \mathrm{e} \mu / \mathrm{g}$, respectively. The $\mathrm{M}-\mathrm{H}$ curves for all the Co-Fe coatings exhibited soft ferromagnetic behaviour with narrow hysteresis loops. It was found that increasing the deposition time also improved the microhardness and magnetic properties of Co-Fe nanocoating, which is much needed for long-life high-coercivity magnetic strip card applications.

\section{Introduction}

Nanoparticles are ultrafine particles with a diameter less than $100 \mathrm{~nm}$ which are fundamental in nanocrystalline materials. These materials exhibit enhancing properties, that is, physical, chemical, and mechanical, resulting from a reduction of grain size and existence of interphase within the grain boundaries of the microstructure $[1,2]$. Electrodeposition is a viable, low-cost process in synthesizing the nanomaterials. It can be used on metals, alloys, polymers, and composites [3]. It can also produce coatings on various substrates requiring higher deposition rates [4]. Electrodeposition is also suitable for any industrial application since it can produce deposits for restricted areas such as tiny parts in machines or any equipment. In electrodeposition, the properties of the nanocrystalline materials can be improved along with their microstructure being controlled by optimizing the operating parameters such as deposition time, $\mathrm{pH}$, bath temperature, direct current density, and electrolytic composition. A diverse range of applications has resulted in the interest for nanoparticles with a wide range of magnetic properties. The study of magnetic properties for softer magnetic materials has gained interest among the researchers due to their potential in the manufacturing of components for microelectromechanical system (MEMS) [5]. In magnetic data storage applications, ferromagnetic nanoparticles with a high coercivity, $H_{c}$, and saturation magnetization, $M_{s}$, is explored for high-density magnetic media such as magnetic recording write heads [68]. The magnetic properties such as $M_{s}$ and $H_{c}$ of soft magnetic materials are very important to be studied in order to evaluate the performance of those soft magnetic materials.

Previous findings have reported on the magnetic properties, $M_{s}$ and $H_{c}$, of Co-Fe alloys in relation to their different alloy compositions in electrolytes [9]. It was found that the values of $M_{s}$ and $H_{c}$ increased linearly with additional contents of Fe. The increase in the $M_{s}$ value was correlated to the crystal structure or phases of the films. The coexistence of both Co-Fe face-centred cubic (FCC) and $\mathrm{Co}-\mathrm{Fe}$ 
body-centred cubic (BCC) phases in the microstructure was possibly caused by higher $M_{s}$ values as the Fe content was increased. Additionally, initial research works reported that, for nanocrystalline materials, there was a large reduction in $M_{s}$ with decreasing grain size [10]. Reference [10] stated a $40 \%$ decrease in $M_{s}$ for nanocrystalline Fe compared to its bulk polycrystalline $\mathrm{Fe}$. It was then concluded that the reduction in $M_{s}$ was attributed to the differences in the magnetic microstructure between nanocrystalline and conventional polycrystalline Fe.

Other previous paper work reviewed the size effects in nanocrystalline metals and alloys made by electroforming, a particular form of electrodeposition [11]. The main size effects in these nanocrystalline materials came from grain size reduction down to less than $5 \mathrm{~nm}$ and down towards the limit of the amorphous structure. Fully dense nanocrystalline metals and alloys have unique microstructures consisting of nanometre-sized crystals and large volume fractions of grain boundary and triple junction defects. It has been shown that several properties such as Young's modulus, thermal expansion, and saturation magnetization were not greatly affected by the presence of high concentrations of these defects. In contrast, properties that depend on the interactions of intercrystalline defects with other structural characteristics such as dislocations, precipitates, and electrons show significant grain size effects. These include hardness, wear resistance, electrical resistivity, and coercivity. In addition, the introduction of structural disorder by grain boundaries and triple junction had an insignificant effect on $M_{s}$ compared with the chemical disorder introduced by additional alloying [12].

The study on the characterization and magnetic properties of electrodeposited Co-Fe alloys with a citrate stabilized sulphate bath was reported elsewhere in relation to the plating conditions and structure parameters [13]. The influence of ammonium citrate dosage, $\mathrm{Fe}$ content, and plating temperature was investigated in relation to magnetic properties. A lower value of $H_{c}$ (10 Oe) was obtained with a low dosage of citrate bath $(2-20 \mathrm{~g} / \mathrm{L})$ due to the low stress in Co-Fe film [14]. The value of $H_{c}$ of the deposited film decreased as the Fe content reached the minimum requirement of approximately 40 atomic\% Fe. However, the $H_{c}$ value slowly rose as the Fe content increased from the minimum content. Similarly, the $H_{c}$ value became greater with increments in the plating temperature. An increase in deposition temperature was most likely due to an increase in deposit grain size, which should have increased the $H_{c}$ value. The $H_{c}$ is directly proportional to the deposited grain size [15]. The composition of Co-Fe film with high $\mathrm{Fe}$ content and less Co content produced the best soft magnetic properties $-H_{c}$ value of $17 \mathrm{Oe}$, which is still acceptable, and an $M_{s}$ value of $2.04 \mathrm{~T}$, which is quite good. The addition of ammonium citrate in the deposition bath was believed to have not sacrificed the magnetic properties of the Co-Fe films. The lowest $H_{c}$ value of $10 \mathrm{Oe}$, which was obtained in a low dosage of ammonium citrate, corresponded to a mixture of FCC and BCC phases with BCC phase denominating. The optimal soft magnetic properties of lower $H_{c}$ and higher $M_{s}$ were produced when both phases, BCC and FCC were codeposited in the Co-Fe microstructure. This is
TABLE 1: Molar concentration of precursors in the electrolyte composition for the synthesis of Co-Fe nanocoating.

\begin{tabular}{lc}
\hline Precursor & Molar concentration, molarity (M) \\
\hline $\mathrm{CoSO}_{4}$ & 0.075 \\
$\mathrm{FeSO}_{4}$ & 0.03 \\
$\mathrm{H}_{3} \mathrm{BO}_{3}$ & 0.4 \\
Sodium saccharine & 0.01 \\
\hline
\end{tabular}

due to the crystallization competition between the BCC and FCC phases which led to the reduction in grain size [15].

Thus, in this research work, the synthesis of Co-Fe nanocoatings using electrodeposition method on stainless steel substrate was reported. The physical and magnetic properties of as-synthesized Co-Fe deposits were investigated in relation to the different deposition times of 30,60 , and 90 minutes.

\section{Experimental Work}

The electrolyte used in the electrodeposition process was a mixture of $\mathrm{CoSO}_{4}, \mathrm{FeSO}_{4}$, and $\mathrm{H}_{3} \mathrm{BO}_{3}$ with an organic additive of saccharine [16]. Table 1 represents the molar concentration of the precursors in the bath composition. The Co-Fe coatings were deposited on the stainless steel substrate while a graphite electrode was used as the anode. A Co-Fe nanocrystalline was deposited with a flow of direct current of density $0.08 \mathrm{~A} / \mathrm{cm}^{2}$. The $\mathrm{pH}$ of the electrolyte was maintained at 3.0. The Co-Fe coatings were formed in 30-, 60- and 90minute deposition times. All the operating parameters such as temperature, $\mathrm{pH}$, direct current density, and deposition time during the electrodeposition process were controlled and maintained. Each experiment was carried out with a freshly prepared solution. The equipment used to test the characteristics of as-synthesized Co-Fe coatings were ULTIMA IV FD $3668 \mathrm{~N}$, X-ray diffractometer (XRD), and JEOL JSM-7600F, a Field Emission Scanning Electron Microscope (FESEM). Hardness of the coating sample was measured with a MITUTOYO MVK-H1, Vickers Microhardness Tester using a load of $200 \mathrm{gm}$. The Alternating Gradient Magnetometer, AGM (Model 2900) was used to determine the magnetic properties of Co-Fe coatings. Figure 1 illustrates the schematic diagram of the electrodeposition process for the synthesis of Co-Fe nanocoating.

\section{Results and Discussion}

3.1. Phase and Crystallographic Structure Characterization. XRD measurements of as-synthesized Co-Fe coatings were carried out from $30^{\circ}$ to $100^{\circ} 2 \theta$ angle. Figure 2 shows the XRD patterns of the electrodeposited Co-Fe coatings at different deposition times of 30,60 , and 90 minutes. The XRD spectrum for the Co-Fe phase reveals the characteristic peaks at $2 \theta$ angles of $45^{\circ}, 65^{\circ}$, and $83^{\circ}$. The peaks correspond to (110), (200), and (211) planes. The Co-Fe phase is identified as the BCC crystal structure with JCPDS no. 10717173. Observing the XRD spectrum for pure cobalt during depositions of 30 and 60 minutes, the Co phase was observed at $2 \theta$ angles 


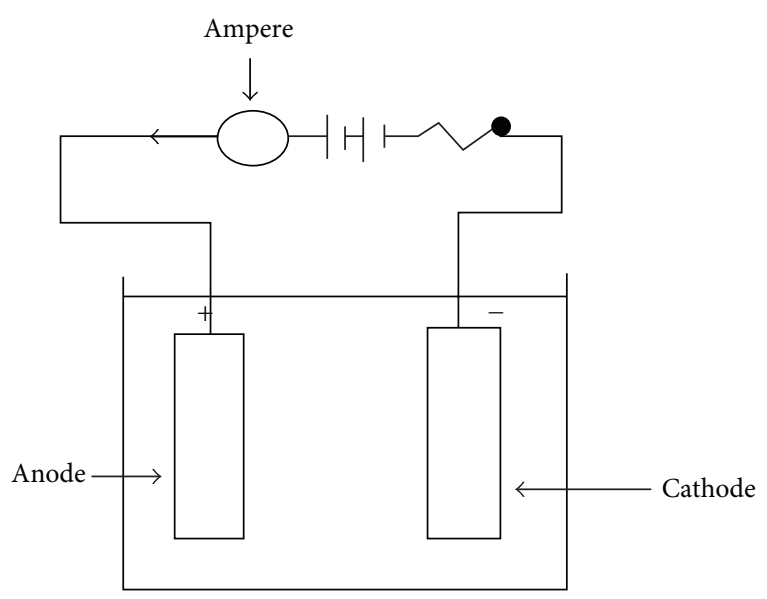

FIGURE 1: Schematic diagram of electrodeposition process.

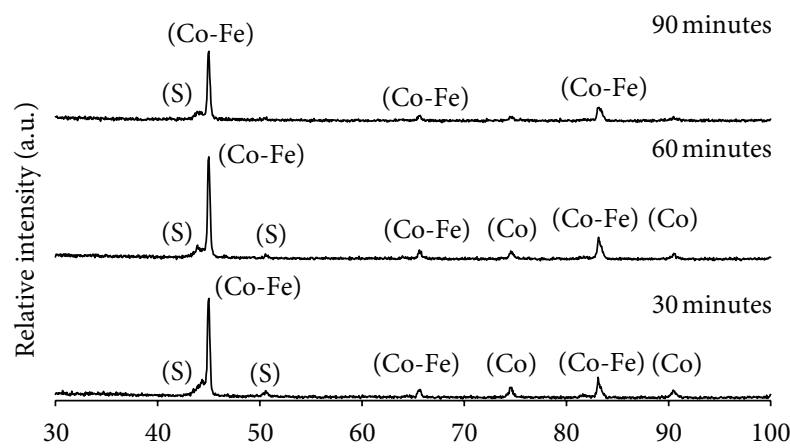

FIGURE 2: X-ray diffraction patterns for three Co-Fe nanocoatings obtained in 30-, 60-, and 90-minute depositions.

of $75^{\circ}$ and $91^{\circ}$ with (110) and (200) planes, respectively. Pure Co phases have the hexagonal close packed (HCP) crystal structure similar with JCPDS no. 50727.

Sulphur (S) peaks were observed in all Co-Fe XRD patterns prepared in three deposition times. The $S$ peaks, which are indexed at $2 \theta$ angles of approximately $44^{\circ}$ and $51^{\circ}$, have the respective corresponding planes of (2210) and (266). S phases have similar orthorhombic crystal structure as with JCPDS no. 80247. As for 90-minute depositions; the $S$ phase reveals a characteristic peak at $2 \theta$ angle of $51^{\circ}$ which disappeared and left only one $S$ phase at $44^{\circ}$.

Saccharine when used as an organic additive in the electrodeposition process of $\mathrm{Co}-\mathrm{Fe}$ nanocoating was the main source for the $S$ element in the microstructure. The incorporation of the $\mathrm{S}$ element in the $\mathrm{Co}-\mathrm{Fe}$ microstructure is believed to have occurred due to the two independent mechanisms. There are saccharine electroreduction and physical incorporation of saccharine molecules during the deposit growth. This incorporation of S elements in the $\mathrm{Co}-\mathrm{Fe}$ microstructure was also reported in [17]. Referring to Figure 2, the peak of pure Co is fully dissolved in a 90minute deposition which showed that the Co-Fe phase was completely formed.
3.2. Surface Morphology Characterization. Figure 3 shows the granular structure of $\mathrm{Co}-\mathrm{Fe}$ coatings in three different deposition times: 30, 60, and 90 minutes. All Co-Fe coatings have dendritic and irregular microstructures. The grains resemble the pine tree-like shape which is known as dendrite, meaning branching. Secondary dendrite arms branch off the primary arm and tertiary arms off the secondary arms, and so forth. The formation of dendrite occurs because the grains are developed in defined planes due to the created crystal lattice [18]. The dendritic microstructure which contains Co-rich content is mostly found in the CoFe coating that was deposited for 30 minutes. This was due to the existence of pure Co phases compared to the other deposition times. This is in agreement with the XRD results. The oriented-attachment mechanism is assumed to have occurred in Co-Fe nanoparticle microstructures which caused the formation of dendritic microstructures [19]. In contrast, the majority of the microstructure area in the 90minute deposition was overwhelmed with the existence of a greater number of irregular grains. These irregular grains are merely grains without a smooth surface because its growth was impeded by contact with another grain or boundary surface. The interface formed between grains is called the grain boundary. The atoms between the grains at the grain boundaries have no crystalline structure and are arranged in a disordered fashion [18]. In conclusion, the presence of irregular grains in the microstructure develops an interface between grain boundaries. Whereas, the grains with dendritic morphology have developed pine tree-like shapes in the microstructure, each with branches that have defined crystal planes.

The average grain sizes decreased from $70.2 \mathrm{~nm}$ to $57.9 \mathrm{~nm}$ with deposition times increasing. It is believed that there is insufficient time for the grains to distribute and therefore leave certain substrate areas empty in the microstructure of Co-Fe nanocoating for 30-minute depositions. The phenomenon has led to the creation of underdeveloped dendritic structures and bigger grain size due to the distribution of the $\mathrm{Co}-\mathrm{Fe}$ atoms being mostly inside the grains compared to the grain boundaries. Agglomeration and grain growth tend to happen in 60-minute depositions as shown in the Figure 3(b). The atoms in the microstructure are fused, combining with each other and producing larger grains. Meanwhile, the irregular grains are smaller in FESEM micrographs of 90minute electrodepositions compared to 30- and 60-minute depositions as shown in Figure 3(c).

In the 90-minute deposition, the Co-Fe atoms have ample time for rearranging themselves in the microstructure as compared to other's deposition times. For the rearrangement of atoms occurring in 90-minute depositions, a large amount of Co-Fe atoms have the opportunity to be located around the grain boundaries with a more complicated and disorderly manner. There are restrictions in their arrangement imposed by the adjacent crystal lattice of disoriented grains in the microstructure [20]. These Co-Fe atoms are arranged with a variety of interatomic spacing differing from boundary to boundary [2]. Furthermore, a greater number of grain boundaries with the highest proportion of atoms inside the boundaries have created an extremely high fractional volume 


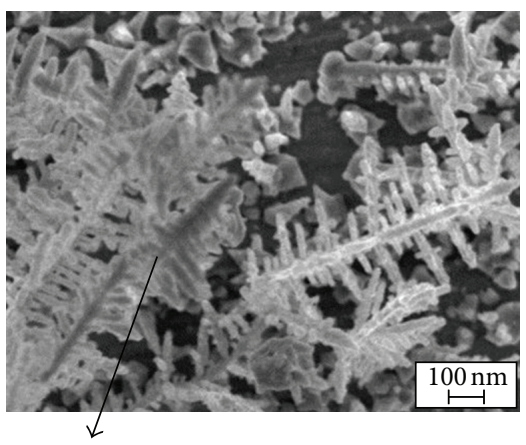

Dendrite shape

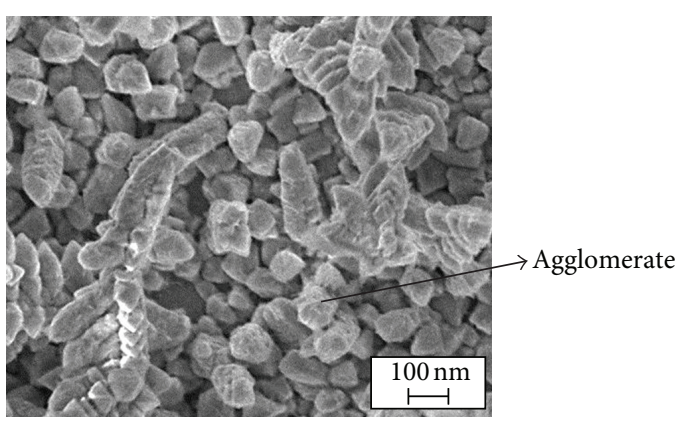

(b)

(a)

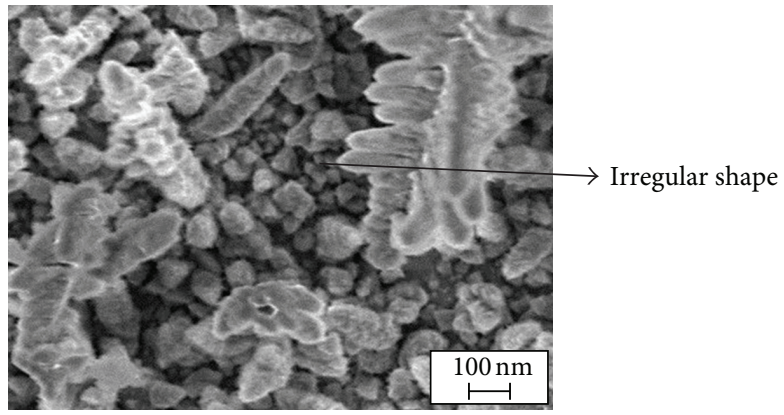

(c)

FIGURE 3: FESEM micrographs of Co-Fe nanocoatings deposited in (a) 30, (b) 60, and (c) 90 minutes.

of grain boundary phases in the Co-Fe microstructure. Therefore, a greater volume fraction of grain boundary phases has developed the fully compact and dense Co-Fe microstructure which has resulted in a reduction in grain size and enhanced properties from the bulk materials. In addition, the Co-Fe microstructure is also believed to be fully completed in this deposition time since there is no presence of pure Co phases in the microstructure as can be seen in the XRD result. As a conclusion, higher proportion of boundary atoms in grain boundaries compared to those inside the grains has created the fully dense and compact microstructure. These high volume fractions of grain boundaries lead to the reduction of grain size.

3.3. Microhardness Measurement. The microhardness measurements were taken from an average of 10 measurements using a $200 \mathrm{gm}$ load. The microhardness values of assynthesized Co-Fe coated samples in 30-, 60-, and 90-minute depositions were 287, 291, and $339 \mathrm{HVN}$, respectively, as shown in Figure 4. The highest microhardness was found at a deposition time of 90 minutes. This was with the smallest grain size $(57.9 \mathrm{~nm})$ and had an existence of structure compactness and grain boundaries in the coating microstructure. This is in parallel with the FESEM result. Moreover, the existence of these grain boundaries prevents the dislocation motion by changing the direction or stopping the movement of the dislocation. Consequently, the change in the dislocated direction movement results in a harder material. This result

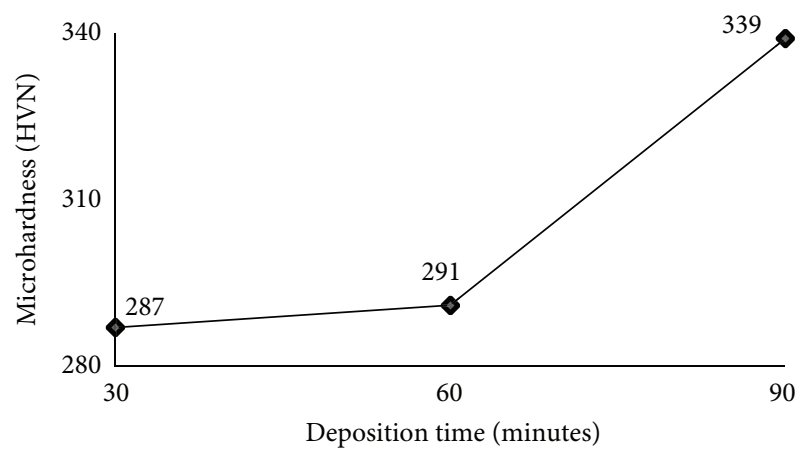

FIGURE 4: The average microhardness of Co-Fe coatings at different deposition times of 30,60, and 90 minutes.

is consistent with [21]. It can be concluded that as the deposition time increases, the average microhardness of the Co-Fe coatings increases.

3.4. Magnetic Properties. Figure 5 shows the magnetic properties of as-synthesized Co-Fe coatings at different deposition times of 30,60, and 90 minutes in $\mathrm{M}-\mathrm{H}$ hysteresis loops. The $\mathrm{M}-\mathrm{H}$ curve of $\mathrm{Co}-\mathrm{Fe}$ coatings passes the origin of the graph. All the M-H curves illustrate that the electrodeposited CoFe coatings exhibit the soft ferromagnetic behaviour due to the presence of narrow and smaller well-defined hysteresis loop areas which can be seen in Figure 4. This similar finding 


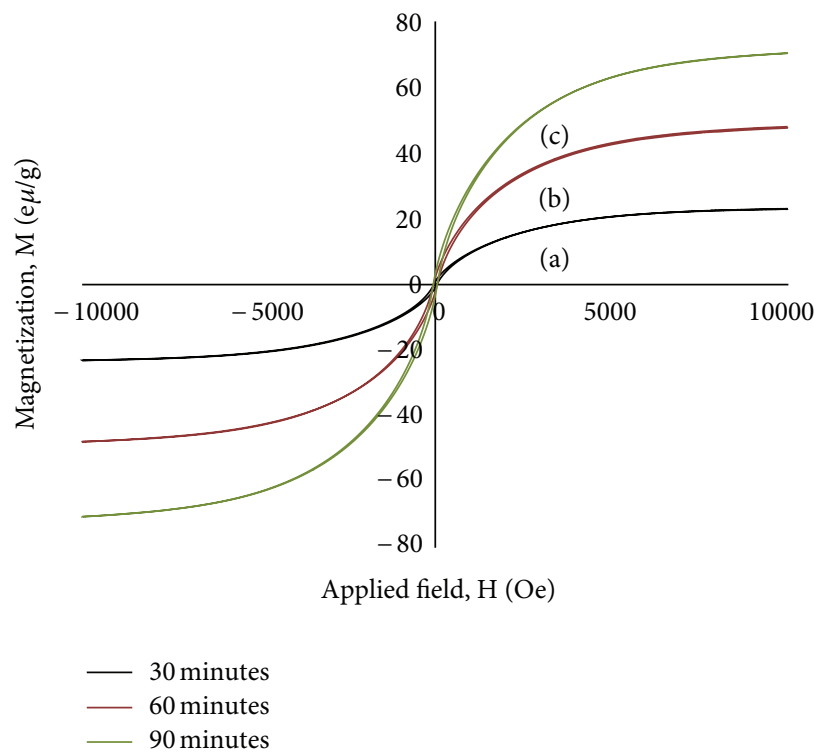

Figure 5: M-H hysteresis loops of Co-Fe nanocoatings prepared in deposition times of (a) 30, (b) 60, and (c) 90 minutes.

TABLE 2: The magnetic properties of electrodeposited Co-Fe coatings in three different deposition times.

\begin{tabular}{lcc}
\hline Deposition time (minutes) & $M_{s}(\mathrm{e} \mu / \mathrm{g})$ & $H_{c}(\mathrm{Oe})$ \\
\hline 30 & 22.99 & 42.56 \\
60 & 47.87 & 52.67 \\
90 & 70.45 & 56.43 \\
\hline
\end{tabular}

was reported in [22]. The Co-Fe nanoparticles in 90-minute depositions show a broader hysteresis loop area compared to the other samples indicated by the higher $M_{s}$ value.

The magnetic properties of electrodeposited Co-Fe coatings for different deposition times are tabulated in Table 2. The $M_{s}$ of Co-Fe coating increases with longer deposition times from 22.99 to $70.45 \mathrm{e} \mu / \mathrm{g}$. This increase in $M_{s}$ value can be explained by the change in grain shape from dendritic to irregular for longer deposition times [5]. The highest proportion of the irregular grains in the 90-minute deposition of the Co-Fe nanocoating developed a compact and fully dense microstructure with smaller grain sizes. The reduced grain size and structure compactness have resulted from a high volume of disorderly arranged atoms in the grain boundaries as compared to the grains themselves. This phenomenon has led to the increase of $M_{s}$ [23]. The formation of a compact dendritic morphology in the crystallized Co-Fe-based alloy was believed to have given an undesirable effect towards the soft magnetic properties [24].

The value of $H_{c}$ was found to be greater with the grain size reducing as deposition times increased. The increase of $H_{c}$ is usually influenced by larger grain sizes [23] but was not consistent with the results of this research. Additionally, based on research work taken from $[25,26]$, low $H_{c}$ was found in the Co-Fe FCC + Co-Fe BCC region. However, in 30-minute depositions, low $H_{c}$ value cannot be related to the creation of combined FCC + BCC phases since Co-Fe phases exhibit pure BCC crystal structures. Therefore, further research should be conducted to investigate this phenomenon of $H_{c}$ increment.

\section{Conclusion}

The Co-Fe phase in the Co-Fe coatings has a BCC crystal structure. Morphology studies reveal that the granular structure of Co-Fe coatings consist of a dendritic and irregular microstructure. The average grain size for all Co-Fe coatings is between $57.9 \mathrm{~nm}$ and $70.2 \mathrm{~nm}$ with the smallest grain size being noted in a deposition time of 90 minutes. The smallest grains produced the highest microhardness of $339 \mathrm{HVN}$. The mechanical properties of microhardness are not affected by the presence of phases in the Co-Fe microstructure. On the other hand, this mechanical property is closely correlated to the reduction in grain size and interactions of intercrystalline defects, that is, grain boundaries with other structural characteristics which include dislocations in the Co-Fe microstructure.

All the Co-Fe coatings show soft ferromagnetic behaviour with narrow hysteresis loops. The $M_{s}$ and $H_{c}$ are increased as the deposition time increases. $M_{s}$ of electrodeposited ferromagnetic materials such as $\mathrm{Co}-\mathrm{Fe}$ nanocoating are generally independent of electroplating conditions. It is only dependent on film compositions (Fe and Co content), phases and crystallographic structures (BCC phase), and surface morphologies (irregular and dendritic structures) in the microstructure. Usually, $H_{c}$ values are directly proportional to the grain size and are affected by the presence of intercrystalline defects of grain boundaries and triple junctions. The best optimal magnetic properties which have reduced $H_{c}$ and higher $M_{s}$ are related to the coexistence of Co-Fe BCC and FCC phases in the microstructure. However, in this research, with the existence of only pure Co-Fe BCC phases, higher $M_{s}$ and $H_{c}$ values were found during depositions times of 90 minutes. 
Effects on grain size reduction are directly proportional to the increase of $M_{s}$ and $H_{c}$. It is suggested that the nanocrystalline Co-Fe coatings prepared at 90 minutes produce better $H_{c}$ and hardness due to smaller grain size.

\section{Acknowledgments}

Financial supports from the Fundamental Research Grant Scheme (600-RMI/FRGS 5/3 (55/2012)) and the Universiti Teknologi Mara (UiTM) Excellent Fund (600-RMI/ST/ DANA 5/3/Dst (373/2011)) and (600-RMI/ST/DANA 5/3/Dst (491/2011)) are gratefully acknowledged.

\section{References}

[1] C. Suryanarayana and C. C. Koch, "Nanocrystalline materialscurrent research and future directions," Hyperfine Interactions, vol. 130, no. 1-4, pp. 5-44, 2000.

[2] C. Suryanarayana, "Nanocrystalline materials," International Materials Reviews, vol. 40, no. 2, pp. 41-64, 1995.

[3] I. Gurrappa and L. Binder, "Electrodeposition of nanostructured coatings and their characterization-a review," Science and Technology of Advanced Materials, vol. 9, no. 4, Article ID 043001, 11 pages, 2008.

[4] U. S. Mohanty, "Electrodeposition: a versatile and inexpensive tool for the synthesis of nanoparticles, nanorods, nanowires, and nanoclusters of metals," Journal of Applied Electrochemistry, vol. 41, no. 3, pp. 257-270, 2011.

[5] N. V. Myung, D. Y. Park, P. T. A. Sumodjo, and B. Y. Yoo, "Development of electroplated magnetic materials for MEMS," Journal of Magnetism and Magnetic Materials, vol. 265, no. 2, pp. 189-198, 2003.

[6] P. Tartaj, M. P. Morales, S. Veintemillas-Verdaguer, T. GonzálezCarreño, and C. J. Serna, "The preparation of magnetic nanoparticles for applications in biomedicine," Journal of Physics D, vol. 36, no. 13, pp. R182-R197, 2003.

[7] H. Chiriac, A. E. Moga, and C. Gherasim, "Preparation and characterization of Co, Fe and Co-Fe magnetic nanoparticles," Journal of Optoelectronics and Advanced Materials, vol. 10, no. 12, pp. 3492-3496, 2008.

[8] X. Liu, P. Evans, and G. Zangari, "Electrodeposited Co-Fe and Co-Fe-Ni alloy films for magnetic recording write heads," IEEE Transactions on Magnetics, vol. 36, no. 5, pp. 3479-3481, 2000.

[9] S. H. Teh and I. I. Yaacob, "Synthesis and characterization of Co-Fe nanocrystalline magnetic films electrodeposited from electrolyte solution containing sodium saccharin," IEEE Transactions on Magnetics, vol. 47, no. 10, pp. 4398-4401, 2011.

[10] H. Gleiter, "Nanocrystalline materials," Progress in Materials Science, vol. 33, no. 4, pp. 223-315, 1989.

[11] U. Erb, "Size effects in electroformed nanomaterials," Key Engineering Materials, vol. 444, pp. 163-188, 2010.

[12] B. Szpunar, U. Erb, K. T. Aust, G. Palumbo, and L. J. Lewis, "The influence of grain-boundary structural disorder on the magnetic properties of nanocrystalline nickel," in Material Research Society Symposium Proceeding, vol. 318, pp. 477-482, 1994.

[13] Y. Zhang and D. G. Ivey, "Characterization of Co-Fe and Co-FeNi soft magnetic films electrodeposited from citrate-stabilized sulfate baths," Materials Science and Engineering B, vol. 140, no. 1-2, pp. 15-22, 2007.
[14] I. Tabakovic, V. Inturi, and S. Riemer, "Composition, structure, stress, and coercivity of electrodeposited soft magnetic CoNiFe films: thickness and substrate dependence," Journal of the Electrochemical Society, vol. 149, no. 1, pp. C18-C22, 2002.

[15] G. Herzer, "Grain size dependence of coercivity and permeability in nanocrystalline ferromagnets," IEEE Transactions on Magnetics, vol. 26, no. 5, pp. 1397-1402, 1990.

[16] G. Fortas, S. Sam, Z. Fekih, and N. Gabouze, "Electrodeposition of CoNiFe alloys on n-type silicon," Materials Science Forum, vol. 609, pp. 207-212, 2009.

[17] J. George, J. Rantschler, S. E. Bae, D. Litvinov, and S. R. Brankovic, "Sulfur and saccharin incorporation into electrodeposited CoFe alloys: consequences for magnetic and corrosion properties," Journal of the Electrochemical Society, vol. 155, no. 9, pp. D589-D594, 2008.

[18] Information on http://www.ndt-ed.org

[19] L. P. Zhu, H. M. Xiao, W. D. Zhang, Y. Yang, and S. Y. $\mathrm{Fu}$, "Synthesis and characterization of novel three-dimensional metallic Co dendritic superstructures by a simple hydrothermal reduction route," Crystal Growth and Design, vol. 8, no. 4, pp. 1113-1118, 2008.

[20] I. A. Ovid'ko, C. S. Pande, and R. A. Masumura, "Grain boundaries in nanomaterials," in Nanomaterials Handbook, Y. Gogotsi, Ed., CRC Press, 2006.

[21] Y. Marita and I. I. Yaacob, "Synthesis and characterization of nickel-iron-silicon nitride nanocomposite," Advanced Materials Research, vol. 97-101, pp. 1360-1363, 2010.

[22] C. K. S. Cheung, Synthesis and microstructural characterization of electrodeposited nanocrystalline soft magnets [Ph.D. thesis], Queen's University, Kingston, Ontario, Canada, 2001.

[23] T. Yokoshima, K. Imai, T. Hiraiwa, and T. Osaka, "Preparation of high-Bs Co-Fe soft magnetic thin films by electrodeposition," IEEE Transactions on Magnetics, vol. 40, no. 4, pp. 2332-2334, 2004.

[24] H. F. Li and R. V. Ramanujan, "Synthesis of Fe-Co based nanomagnetic materials," Transactions of the Indian Institute of Metals, vol. 58, no. 6, pp. 965-970, 2005.

[25] T. Osaka, T. Sawaguchi, F. Mizutani, T. Yokoshima, M. Takai, and Y. Okinaka, "Effects of saccharin and thiourea on sulfur inclusion and coercivity of electroplated soft magnetic CoNiFe film," Journal of the Electrochemical Society, vol. 146, no. 9, pp. 3295-3299, 1999.

[26] T. Osaka, "Electrodeposition of highly functional thin films for magnetic recording devices of the next century," Electrochimica Acta, vol. 45, no. 20, pp. 3311-3321, 2000. 

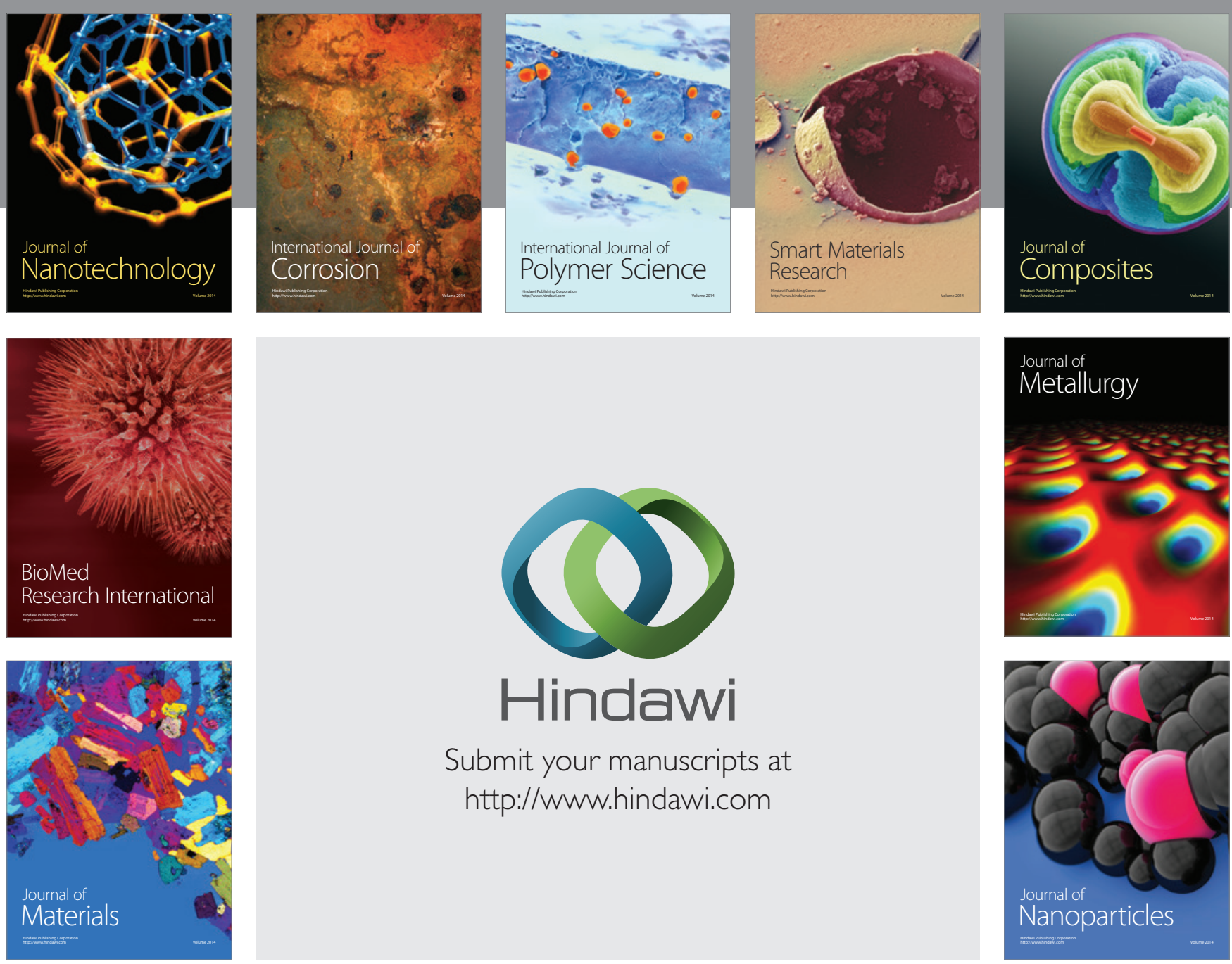

Submit your manuscripts at http://www.hindawi.com
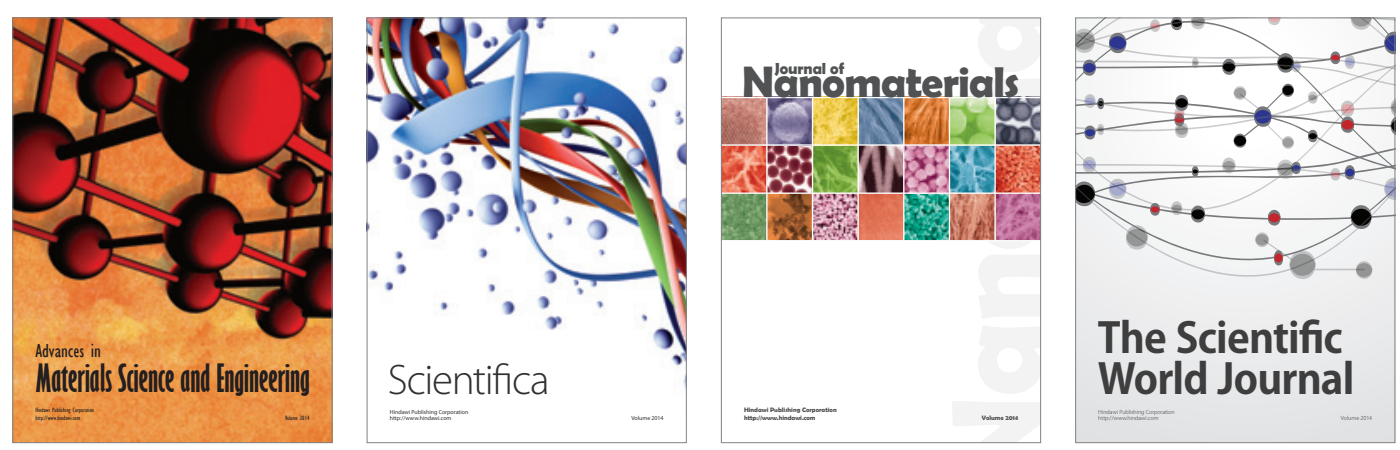

\section{The Scientific World Journal}
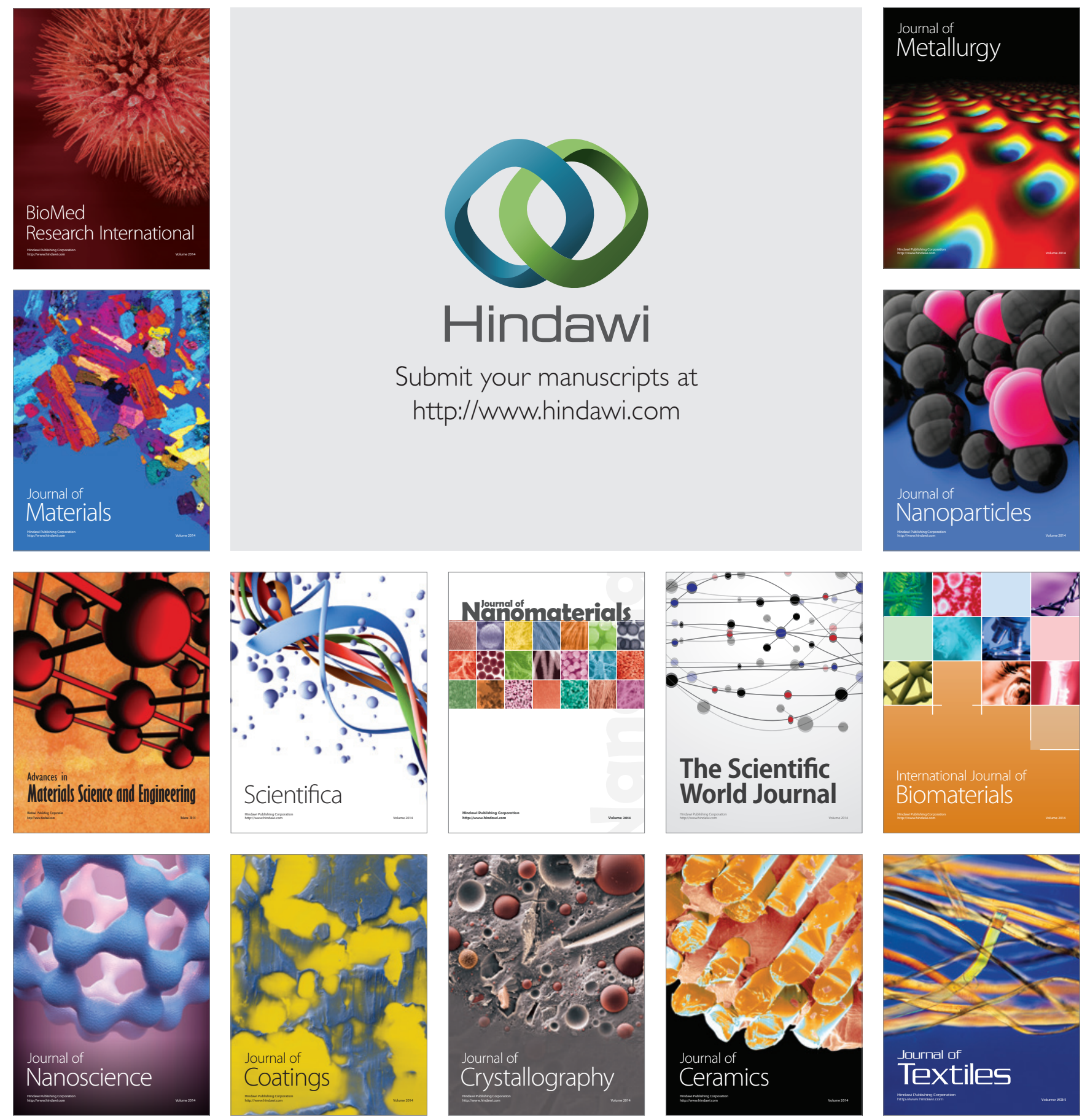